\title{
Histologic Study on the Sites of Side-Arm Branching of the Retinal Arterioles in Spontaneously Hypertensive Rats (SHR)
}

\author{
Tadao Sekino and Kimiho IrINodA
}

Existence of protuberances into lumina has been pointed out as one of histologic changes in retinal arterioles of spontaneously hypertensive rats (referred to hereafter as SHR) up to now. In this study, the author examined the retinal arterioles of SHR by serial sections to clarify sites where the protuberances existed and roles that they played in the retinal arterioles, and ascertained that the protuberances existed at the sites of side-arm branching of the arterioles. Histologic findings of the protuberances at the sites of side-arm branching and the mechanism by which blood circulation is regulated there were discussed.

Eight SHRs, 1.5 to 17 months old, were used for this study. After enucleation, the retinas were dissected into small pieces containing retinal arterioles in $4 \%$ glutaraldehyde. The retinal specimens were prefixed in $4 \%$ glutaraldehyde and postfixed in $1 \% \mathrm{OsO}_{4}$ and then dehydrated in series of graded ethanol. The retinal specimens were embedded in Epon 812 resin and sectioned with a LKB Ultrotome. The sections were stained with toluidine blue for viewing with a light microscope. Thin sections for electron microscopy were stained with lead acetate and examined with a HITACHI HS-8 or HU-12A electron microscope.

Five normotensive Wistar-Kyoto rats, 6 to 24 months old, were examined as controls by the same method as the SHR.

Blood pressure was measured indirectly by tail plethysmography.

All rats were fed with a pellet diet (Oriental MF).

Systolic blood pressure of a 1.5-month-old SHR was $116 \mathrm{mmHg}$. Those of the SHRs older than 4 months ranged from $192 \mathrm{mmHg}$ to $224 \mathrm{mmHg}$.

The control rats showed normal blood pressures ranging from $114 \mathrm{mmHg}$ to $144 \mathrm{mmHg}$.

In all cases of SHR examined, annular protuberances into the lumina, which were called annuli, were found at the sites of side-arm branching of the retinal arterioles in observation by serial sectioning. The annuli completely encircled and narrowed the orifices of the branches. These protuberances appeared 5 to $25 \mu$ before the branching points and disappeared 5 to $15 \mu$ after encircling the orifices of the branches. The protuberances showed marked decrease in toluidine blue staining.

In the control rats, annular protuberances were found as well as the SHRs at the sites of side-arm branching of the arterioles.

By electron microscopic observation, a layer of endothelial cells, remarkably thickened and reduplicated basal lamina that showed low electron density in places, and island-shaped and zonal cytoplasms surrounded by thickened basal lamina in subendothelial layers were observed at the annuli in both the SHRs and the control rats. One to 3 layers of smooth muscle cells that were island-shaped in places were

From the Department of Ophthalmology, Hirosaki University School of Medicine, Hirosaki. 
observed in the outermost portion at the annuli. The endothelial cells stretched long cytoplasmic processes into the basal lamina and often possessed nuclei bulging into the lumina and were occasionally very thin in part. The internal elastic lamina was splitted among the cytoplasms in the subendothelial layer and fragmented or disappeared. The cytoplasms, in the luminal portion of the subendothelial layers, generally showed irregular shapes and arrangements and were similar to the cytoplasms of the endothelial cells, and appear to be part of the endothelial cell processes or be separated from endothelial cells. On the other hand, those in the basal portion often showed annular arrangements and possessed a few filaments resembling myofilaments, and may be of origin from smooth muscle cells. The cytoplasms in the subendothelial layers were frequently in contact with each other or the endothelial cells without interception of the basal lamina. Cellular debris was found in the basal lamina at the annuli even in a 1.5-month-old SHR and a 6-monthold control rat. The basal lamina contained a larger amount of cellular debris and was thicker in the SHRs with long duration of hypertension than in the aged control rats.

Also in human, cat, mouse, dog and pig,, annuli have been observed at sites of side-arm branching of retinal arterioles. In regard to the roles that the annuli play, a few assumptions have been given. It was suggested that they probably did not actively contract but could constrict the lumen by swelling. Besides, the peculiar structure of the sphincter was observed and it was suggested that the root of branch regulated the blood circulation by changing the tone of component cells.

This electron microscopic examination revealed nuclei of the endothelial cells bulging into the lumina, remarkably thickened basal lamina and island-shaped and zonal cytoplasms at the annuli of the side-arm branching sites of the retinal arterioles in the SHRs and the control rats. The orifice of the branch is narrowed by bulging of the subendothelial layer and by the existence of the endothelial cell nuclei. However, the peculiar contractive structure was not observed. Namely, it is assumed that the arteriolar annuli at the sites of side-arm branching serve to temper and regulate the blood circulation from the trunk by narrowness at the entrances of the branches without the function of sphincters in the SHRs and Wistar-Kyoto rats. 